\title{
Air Traffic Control Approach Procedural Separation Assessment Mode
}

\author{
TANG Wei-zhen Assoc. Prof \\ Air Traffic Management College, Civil Aviation Flight University of China \\ Guanghan Sichuan 618307, China \\ Email:tangweizheng@sina.com
}

\begin{abstract}
In recent years, with the rapid increase of air traffic flow, fight conflict have become more and more serious. So it is very necessary to study mid-air collision. The purpose of the paper is to establish the mode of approach procedural separation assessment. The degree which approach separation is been affected by all the factors has been worked out. The distance at which the pilot may report to the ATC (air traffic controller )form the radio blind area center when the aircraft over flying the VOR/NDB and the number of the report at the distance is submit to normal distribution has been approved. The separations have been decided by using the probability and the mathematics methods. According to the relationship of each factor the component of the mode has been decided and the mode has been build up. The mode of approach procedural separation assessment has been tested and extended.
\end{abstract}

Key words : approach procedural separation; radio blind area; normal distribution ; safety assessment; Separation standard

\section{INTRODUCTIONS}

separation is the standard implementation of air traffic control and the basis in order to ensure safety, make full use of spare resources, save fuel and reduce direct operating costs, more and more emphasis on separation international study abroad early in the 20th century, the sixties and seventies he started to reduced separation standards airspace safety assessment studies . This research work is mainly of two kinds. One class to the UK PG Reich established in 1964 based on the theory of aircraft collision model for parallel route systems in the longitudinal, lateral, vertical collision risk modeling respectively study ${ }^{[1]}$. Another theory is based on Rice 's level will start to cross . Both theories are considered first two risk of collision between aircraft, and then gives control of the region under a certain separation of collision risk, which in practical applications in both airspace safety assessment .

1999 Northern Air Traffic Control Research Center, Li Jin, Wang Yingxun and others on parallel routes Reich collision risk model is a preliminary study ${ }^{[2]}$, and modeling are analyzed, but only for the analysis of parallel routes, and is not decomposition to the lateral, longitudinal and vertical directions are considered. 2001 , Professor XuXiaohao Wang Xin led the probabilistic methods used Lyle.D.Filkins on the assumption that the longitudinal and lateral and vertical directions independently of each other, under the premise were analyzed longitudinal, lateral and vertical collision risk issues $^{[2]}$.

Separation starting from the current regulations, a comprehensive analysis of tolerance navigation station, route and airspace structure, flying the human factor and many other factors influence the program control interval [1] , solving approach procedures control the aircraft during a specific separation Why should meet problem . Research approach procedures affecting factors intervals and related factors on the relationship between the impact of the approach procedure interval case, the probability of occurrence of each factor to determine the level of security required to meet certain conditions, by demonstrating the basis for determining the appropriate separation, for the existing air traffic control interval approach procedures provide theoretical support security.

\section{SEPARATION OF FACTORS AND THEIR IMPACT ON PROGRAM ANALYSIS}

\section{A. Tolerance navigation station}

Civil Aviation of China and the world is currently used by civil aviation ground navigation stations are to VOR 
(VHF omnidirectional beacon) and NDB (non-directional beacon) based ${ }^{[3]}$. Rely on airborne aircraft intercepted the signal can be done to the station or back- stage flight, enabling navigation, the aircraft altitude of 10,000 meters is still able to fly along the planned route . However, both the VOR or NDB, because the signal cannot reach their own headspace a cone-shaped area, forming a blind spot, known as headspace blind spot. Thus aircraft VOR and NDB navigation leap empty stage unable to receive navigation signals, so the pilot cannot determine the aircraft flew over the navigation table empty exact moment. VOR and NDB headspace blind, also called cone effect area, is a radio station for the apex of the inverted cone ${ }^{[4]}$, the radius $(Z)$ based on the real aircraft radio leap height (h) and semi- cone angle (a) Identification, $\mathrm{Z}=\mathrm{h} \cdot \tan \alpha$ formula, VOR ( VHF omnidirectional beacon ) $\alpha$ size is $50{ }^{\circ}, \mathrm{NDB}$ ( non-directional beacon) a size of $40^{\circ}$.

Assuming a flight altitude of $6600 \mathrm{~m}$, then for NDB ( non-directional beacon ), its diameter headspace blind : $=2 \times 6600 \times \tan 40^{\circ} \approx 11076.12(\mathrm{~m})$; empathy for the VOR ( VHF omnidirectional beacon ), which blind headspace diameter $:=2 \times 6600 \times \tan 50^{\circ} \approx 15731.15(\mathrm{~m})$.
From the calculation results, the pilots on the ground navigation empty stage what position report over Taiwan is a key factor in the decision aircraft spacing. For the following reasons :

Because navigation station tolerance reasons, resulting in the pilot reported the aircraft over station interval timing error on the flight size has a very big impact, is the development gap is an important factor to consider.

\section{B. aircraft wake turbulence}

In the wake of aircraft, including the propeller wake flow generated, flaps and fuselage generated turbulence, jet engine exhaust gases as well as the formation of the jet stream vortices ${ }^{[5]}$, which are affected in varying degrees, followed by of the aircraft, where the greatest impact on the trailing aircraft, mainly the formation of vortices trailing vortex. So wake sometimes specifically refers to the formation of vortices trailing vortex ${ }^{[5]}$. Thus during the flight, after the aircraft must maintain a safe distance, so the aircraft wake turbulence is affecting aircraft spacing is another important factor.

Table 1 provides an aircraft wake turbulence separation

\begin{tabular}{|c|c|c|c|c|c|c|c|}
\hline & \multicolumn{7}{|c|}{ Following aircraft } \\
\hline \multirow{5}{*}{ 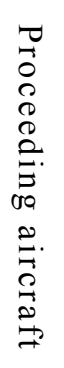 } & $\begin{array}{c}\text { Aircraft } \\
\text { Type }\end{array}$ & Lightweight & Medium & Heavy & Lightweight & Medium & Heavy \\
\hline & Lightweight & 3 & 3 & 3 & 98 & 74 & 74 \\
\hline & Medium & 4 & 3 & 3 & 138 & 74 & 74 \\
\hline & Heavy & 6 & 5 & 4 & 167 & 114 & 94 \\
\hline & & \multicolumn{3}{|c|}{$\begin{array}{l}\text { Minimum distance separation } \\
\qquad(\mathrm{NM})\end{array}$} & \multicolumn{3}{|c|}{$\begin{array}{l}\text { The minimum time separation } \\
\text { (s) }\end{array}$} \\
\hline
\end{tabular}

\section{C.Other secondary factors}

Flight crew and control personnel reaction time; equipment occupancy time delay and the impact of information dissemination. According to the provisions of the program design principles, flight crew reaction time is $0-6 \mathrm{~s}$, and thus reflects the author's time to determine the maximum value of $6 \mathrm{~s}$, flight crew and control personnel reaction time is defined as $6 \mathrm{~s}$. As the rapid development of science and technology today, Air Traffic Management systems and instruments used in aircraft systems continue to improve accuracy, the device delays are mostly subtle level, the time is rounded up, I eventually identified as transceiver system $1 \mathrm{~s}$, equipment of the error caused by $2 \mathrm{~s}$. Signal propagation time error by double maximum height value $12000 \mathrm{~m}$, speed at the speed of light : $300,000 \mathrm{~km} /$ second terms, the maximum error caused by signal propagation is : $12000 \mathrm{~m} /$ $(300000000 \mathrm{~m} / \mathrm{s})=0.00004 \mathrm{~s}$, also rounded up 
the signal obtained Communication errors caused to $1 \mathrm{~s}$. Affect the program spaced intervals factors caused some minor errors in the total time: $6 s+6 s+1 s+1 s+1 s=15 s$.

\section{BUILT TOUCH CONTROL INTERVAL ASSESSMENT}

\section{A. underlying assumptions}

In order to avoid overly complicated and trivial mathematical calculations, the subject right approach to do a certain interval of model simplification, only consider the same route with a high degree intervals situation. By analyzing the difference in speed of the aircraft, the subject of the approach to be established between assessment models make the following assumptions:

- consider the aircraft to a constant before and after the two aircraft ;

- consider the same route aircraft around two different heights ;

- consider the independent route, without regard to the specific airspace structure.

\section{B.Assessment touch control interval construction}

The subject will be discussed at an important variable in the form of the probability density function is as follows:

$\mathrm{f}(\mathrm{x})=\frac{1}{\sqrt{2 \pi} \sigma} e^{-\frac{(x-\mu)^{2}}{2 \sigma^{2}}}-\infty<x<\infty$

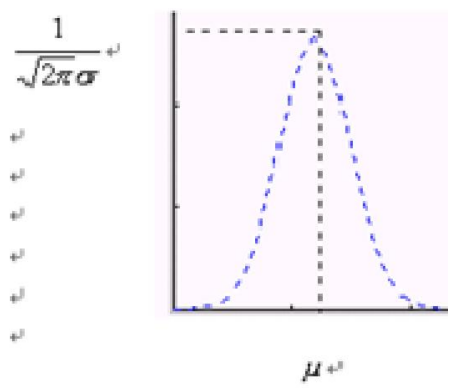

Figure 1 Normal probability density curve

Where is a constant, and said obey this probability density $\mathrm{X}$ is normally distributed or Gaussian distribution, denoted by, probability density function shown at left. When a variable is known to follow a normal distribution or according to the data collected after fitting is normally distributed, the left can be expressed according to the relationship between the calculated ${ }^{[7]}$.

Individual aircraft height errors follow a normal distribution, height keeping errors are mainly distributed in between -400 feet to 400 feet, 300 feet higher than the error small. By a, may draw its follow a normal probability density curve, the probability density function is:

$\mathrm{f}(\mathrm{x})=\frac{1}{\sqrt{2 \pi} \sigma} e^{-\frac{(x-\mu)^{2}}{2 \sigma^{2}}}$

The method can be demonstrated by panning, navigation table empty reported that over station aircraft deviated from its blind spots headspace center and the number of aircraft and the aircraft deviated from its assigned altitude deviation of distance and the number of aircraft is consistent with a normal distribution ${ }^{[7]}$. Theoretically aircraft headspace blind equal distance on both sides of the center where the probability of reporting over station is the same, for the sake of accuracy, so that the value is 0 , that is, the station reported positional deviation of the probability density function is:

$f(x)=\frac{1}{\sqrt{2 \pi} \sigma} e^{-\frac{x^{2}}{2 \sigma^{2}}}$

$\sigma=101.414, \mathrm{X}$ represents the distance from the center.

Program design, program design principles to aircraft deviated from when the probability is less than the protected area that is to meet the safety requirements [8], where the requirement to apply this probability is calculated when the aircraft deviated from its specified altitude deviation probabilities corresponding offset distance too.

By the formula (2) can be obtained:

$\mathrm{F}(\mathrm{x})=\int_{-\infty}^{x} \frac{1}{\sqrt{2 \pi} \delta} e-\frac{(x-\mu)^{2}}{2 \delta^{2}}=10^{-7}$ 
$\mu=-9.281, \sigma=101.414$

From reference ${ }^{[7]}$ Schedule look-up table

$x_{1}^{\prime}=3.8, x_{2}^{\prime}=3.9, x=x^{\prime} \bullet \delta+\mu$, So

$x_{1}=376.0922, x_{2}=386.2336$,

$P\left(x_{1}\right)=0.9999, \quad P\left(x_{2}\right)=1$; Since the

probability of seeking is $10^{-7}$, Therefore, we must interpolate between the two operations, the operation is as follows:

$x=x_{2}-\left(x_{2}-x_{1}\right) \cdot \frac{10^{-7}}{1-0.9999}=386.2235$

That is, the aircraft deviated from its assigned altitude 386.2235 feet probability is 10

Our approach control airspace ceiling of $6000 \mathrm{~m}$, the lower limit for the tower control zone limit, typically $900 \mathrm{~m}$. For safety reasons, and in order to ensure the adaptability separation when creating a model of the subject will be calculated $6000 \mathrm{~m}$ altitude navigation station headspace blind range, while taking into account the VOR (VHF omnidirectional beacon) and NDB (non-directional beacon ) headspace blind a different half cone angle, VOR station a size of $50^{\circ}$, NDB sets a size of $40^{\circ}$. Also for safety reasons, and in order to ensure the adaptability interval when creating a model of the subject will be VOR (VHF omnidirectional beacon) a size of 50 calculation. So blind navigation station headspace radius

$\mathrm{Z}=6000 \times \tan 50^{\circ} \approx 7150.5216 \mathrm{~m}$

According discussed above, aircraft navigation station at a distance of headspace at the blind center $7150.5216 \mathrm{~m}$ probability reported over station

Formula (2) in the probability of the offset distance of $10^{-3.5}=0.0003162$

$\mathrm{F}(\mathrm{x})=\int_{-\infty}^{x} \frac{1}{\sqrt{2 \pi} \delta} e-\frac{(x-\mu)^{2}}{2 \delta^{2}}=10^{-3.5}$ $\mu=-9.281, \quad \sigma=101.414$

From reference ${ }^{[7]} \quad$ Schedule look-up table $x_{1}^{\prime}=3.3, x_{2}^{\prime}=3.4$, $x=x^{\prime} \bullet \delta+\mu$, So $x_{1}=325.3852$, $x_{2}=335.5266$ $P\left(x_{1}\right)=0.9995, P\left(x_{2}\right)=0.9997$; Since the probability of demand is $10^{-3.5}$, so for the two interpolation operation, operation is as follows:

$x=x_{2}-\left(x_{2}-x_{1}\right) \cdot \frac{10^{-3.5}}{0.9997-0.9995}=321.1866$

the aircraft deviated from its assigned altitude

321.1866 feet probability is $10^{-3.5}$ 。

Assuming the aircraft to deviate from the navigation station at $\mathrm{X}$ headspace blind reported over station probability is $10^{-3.5}$, by proportional correspondence can be obtained: $\frac{x m}{7150.5216 m}=\frac{321.1866 f t}{386.2235 f t}$ Solve for $\mathrm{x}=$ 5946.4318

aircraft navigation station at a distance of headspace blind Center 5946.4318 reported over station probability is $10^{-3.5}$, that two aircraft simultaneously at a distance of blind navigation station headspace center 5946.4318 reported over station probability is. ${ }^{-7}$ Therefore, to meet the two aircraft collision probability model under the condition of less than or equal to consider blind navigation station headspace range of $2 \times 5946.4318=11892.8637$.

\section{SPECIFY THE SEPARATION VALIDATION APPROACH PROCEDURES}

Approach control airspace, flying the same speed in the same direction aircraft flying at 
different heights within the same corridor, and with navigation equipment, the minimum interval of $5 \mathrm{~min}$. Navigation station tolerance separation, and its size is:

$\underset{\text { tolerance range }}{\text { aircraft speed }}=\frac{12132.1637 \mathrm{~m}}{400 \mathrm{~km} / \mathrm{h}}=\frac{12132.1637 \mathrm{~m}}{\left(\frac{400000}{3600}\right) \mathrm{m} / \mathrm{s}} \approx 109.1895 \mathrm{~s}$

Ceil was 110s; Minimum wake turbulence separation 167s; Secondary factor separation $15 \mathrm{~s}$.

Therefore, the interval of the model to meet the conditions and limitations under the assumption that subjects met the separation: $110 s+167 s+15 s=292 s 4.8667 m i n$.

Can be drawn from the results clearly established approach the subject separation evaluation model is consistent with the current separation. Moreover, if the model is assessed based on time separation, the end result will make the appropriate treatment, such as the final provisions of the separation $4.8667 \mathrm{~min} 5 \mathrm{~min}$, which end with the current separation maintained a high degree of consistency, to illustrate the topic separation approach established evaluation model is reasonable.

\section{CONCLUSIONS}

$A$. In the right conditions affecting procedural control air traffic control many factors separation

\section{REFERENCES}

[1] Wang Dahai, Yang Jun , Yu Jiang . Flight principle [M].Chengdu : Southwest Jiao tong University Press, 2004:212 215

[2] should Aileen, XuXiaohao Airspace dangerous side collision REICH Model Study [J]. China Civil Aviation College, 2002

[3] Liu Huiying, air traffic management system Introduction [M]. Beijing : National Defense Industry Press, 2002

[4] Zhu on behalf of Wu, Heguang Qin . Visual and instrument flight procedure design [M]. based on the analysis, it was identified that the relationship between factors and established a mathematical model of the existing approach procedures conducted a safety control separation assessment, and use this model to the existing intervals security verification.

$B$.flight separation standard safety assessment studies in the field of civil aviation is still its infancy, there is no mature theory can learn from the study is only tentatively to improve the theory abroad to meet domestic standards of safety assessment separation, the separation standard reduction or re-enactment provides a theoretical basis .

C.Research model takes into account the main factors that blind navigation station headspace range varies with altitude while flying as high altitude areas, the air is less dense, and the ceiling of the aircraft near the aircraft performance will change. Another change in the speed of the aircraft, and the table with the airspeed velocity differences will also produce a certain separation to determine the impact. Considering these factors and then to regional safety margin separation to be considered, the model can also be extended interval evaluation model for regional programs.

Chengdu : Southwest Jiao tong University Press, 2004:12

[5] FengQingchuan . Aerodrome control [M]. Chengdu : Southwest Jiao tong University Press, 2004:67 69

[6] Zhao Yuan. Several new air traffic safety and reliability estimation theory [D]. Beijing : Northern Jiaotong University Press, 1997

[7] Sheng sudden , Shyh- one thousand Pancheng Yi . Probability Theory and Mathematical Statistics ( second edition ) [M]. Beijing : Higher Education Press, 1989:371 